\title{
An Accurate Numerical Solution for the Modified Equal Width Wave Equation Using the Fourier Pseudo-Spectral Method
}

\author{
Hany N. Hassan \\ Department of Basic Engineering Sciences, Benha Faculty of Engineering, Benha University, Benha, Egypt \\ Email: h_nasr77@yahoo.com
}

Received 7 April 2016; accepted 10 June 2016; published 13 June 2016

Copyright (C) 2016 by author and Scientific Research Publishing Inc. This work is licensed under the Creative Commons Attribution International License (CC BY). http://creativecommons.org/licenses/by/4.0/ c) (i) Open Access

\begin{abstract}
In this study, the numerical solution for the Modified Equal Width Wave (MEW) equation is presented using Fourier spectral method that use to discretize the space variable and Leap-frog method scheme for time dependence. Test problems including the single soliton wave motion, interaction of two solitary waves and interaction of three solitary waves will use to validate the proposed method. The three invariants of the motion are evaluated to determine the conservation properties of the generated scheme. Finally, a Maxwellian initial condition pulse is then studied. The $L_{2}$ and $L_{\infty}$ error norms are computed to study the accuracy and the simplicity of the presented method.
\end{abstract}

\section{Keywords}

The Modified Equal Width Wave Equation, Fourier Pseudo-Spectral Method, Solitary Waves, Fast Fourier Transform

\section{Introduction}

Discretization using finite differences in time and spectral methods in space has proved to be very useful in solving numerically non-linear Partial Differential Equations (PDEs) describing wave propagation. The Korteweg de Vries (KdV) equation is one famous example to which such combined schemes have been applied efficiently to analyze efficiently unidirectional solitary wave propagation in one dimension [1]-[3]. In [4] [5] the combination of spectral methods and finite differences is applied to well-known nonlinear PDE of the Boussinesq type which admits bidirectional wave propagation, has closed form solitary wave solutions and like the $\mathrm{KdV}$ is completely integrable in one space dimension. Also, the combination of spectral methods and leap frog 
is applied to the Regularized Long Wave (RLW) equation [6]. In this paper, a combination of spectral method and leap frog is applied to the modified equal width wave equation. The modified equal width wave equation based upon the Equal Width Wave (EW) equation [7] [8] which was suggested by Morrison et al. [9] is used as a model partial differential equation for the simulation of one-dimensional wave propagation in nonlinear media with dispersion processes. This equation is related with the Modified Regularized Long Wave (MRLW) equation [10] and modified Korteweg-de Vries (MKdV) equation [11]. All the modified equations are nonlinear wave equations with cubic nonlinearities and all of them have solitary wave solutions, which are wave packets or pulses. These waves propagate in non-linear media by keeping wave forms and velocity even after interaction occurs. Few analytical solutions of the MEW equation are known. Thus numerical solutions of the MEW equation can be important and comparison between analytic solutions can be made. Geyikli and Battal Gazi Karakoc, [12] [13] solved the MEW equation by a collocation method using septic B-spline finite elements and using a Petrov-Galerkin finite element method with weight functions quadratic and element shape functions which are cubic B-splines. Esen applied a lumped Galerkin method based on quadratic B-spline finite elements which have been used for solving the EW and MEW equations [14] [15]. Saka proposed algorithms for the numerical solution of the MEW equation using quintic B-spline collocation method [16]. Zaki considered the solitary wave interactions for the MEW equation by collocation method using quintic B-spline finite elements [17] and obtained the numerical solution of the EW equation by using least-squares method [18]. Wazwaz investigated the MEW equation and two of its variants by the tanh and the sine-cosine methods [19]. A solution based on a collocation method incorporated cubic B-splines is investigated by and Saka and Dag [20]. Variational iteration method is introduced to solve the MEW equation by Lu [21]. Evans and Raslan [22] studied the generalized EW equation by using collocation method based on quadratic B-splines to obtain the numerical solutions of a single solitary waves and the birth of solitons. Hamdi et al. [23] derived exact solitary wave solutions of the generalized EW equation using Maple software. Esen and Kutluay studied a linearized implicit finite difference method in solving the MEW equation [24]. Karakoç and Geyikli [25] solved the MEW equation numerically by a lumped Galerkin method using cubic B-spline finite elements. The modified equal width wave equation has the normalized form [9]

$$
U_{t}+3 U^{2} U_{x}-\mu U_{x x t}=0,
$$

where $\mu$ is a positive parameter and the subscripts $x$ and $t$ denote differentiation, when solved analytically, within an infinite region with physical boundary conditions $U \rightarrow 0$ as $|x| \rightarrow \infty$. In this study, boundary conditions are chosen from

$$
U(a, t)=0, U(b, t)=0, t>0
$$

and the initial condition

$$
U(x, 0)=f(x), a \leq x \leq b
$$

where $f(x)$ is a localized disturbance inside the considered interval. We investigate the numerical solution of the MEW equation using the Fourier Leap-Frog methods. The proposed method is validated by studying the motion of a single solitary wave, development of interaction of two positive solitary waves and development of three positive solitary waves interaction for the MEW Equation (1).

\section{Analysis the Proposed Method}

For the numerical treatment, the spatial variable $x$ of Equation (1) is restricted over an interval $a \leq x \leq b$. In this study, consider the MEW Equation (1) with the boundary conditions in Equation (2). A numerical method is developed for the periodic initial value problem in which $U$ is a prescribed function of $x$ at $t=0$ and the solution is periodic in $x$ outside a basic interval $a \leq x \leq b$. For most of the problems considered, interval may be chosen large enough so the boundaries do not affect the wave interactions being studied. Equation (1) can be written as

$$
V_{t}=-3 U^{2} U_{x},
$$

where

$$
V=U-\mu U_{x x} .
$$

For ease of presentation the spatial period $[a, b]$ is normalized to $[0,2 \pi]$, with the change of variable 


$$
x \rightarrow \frac{2 \pi}{b-a}(x-a)
$$

Let $L=b-a$. Thus, Equations (4) and (5) become

$$
\begin{aligned}
& V=U-\left(\frac{2 \pi}{L}\right)^{2} \mu U_{x x}, \\
& V_{t}=-3\left(\frac{2 \pi}{L}\right) U^{2} U_{x},
\end{aligned}
$$

$U(x, t)$ is transformed into Fourier space with respect to $x$, and derivatives (or other operators) with respect to $x$. This operation can be done with the Fast Fourier transform (FFT). Applying the inverse Fourier transform $\frac{\partial^{n} U}{\partial x^{n}}=F^{-1}\left\{(i k)^{n} F(U)\right\}, n=1,2, \cdots$ with $n=1$ and $n=2$. The Equations (6) and (7) become

$$
\begin{aligned}
& V=U-\left(\frac{2 \pi}{L}\right)^{2} \mu F^{-1}\left\{-k^{2} F(U)\right\}, \\
& V_{t}=-3\left(\frac{2 \pi}{L}\right) U^{2} F^{-1}\{i k F(U)\} .
\end{aligned}
$$

In practice, we need to discretize Equations (6) and (7). For any integer $N>0$, consider $x_{j}=j \Delta x=\frac{2 \pi j}{N}$, $j=0,1, \cdots, N-1$. Let $U(x, t)$ be the solution of Equations (8) and (9). Then, we transform it into the discrete Fourier space as

$$
\widehat{U}(k, t)=F(U)=\frac{1}{N} \sum_{J=0}^{N-1} U\left(x_{j}, t\right) \mathrm{e}^{-i k x_{j}},-\frac{N}{2} \leq k \leq \frac{N}{2}-1 .
$$

From this, using the inversion formula, we get

$$
U\left(x_{j}, t\right)=F^{-1}(\widehat{U})=\sum_{k=-N / 2}^{N / 2-1} \widehat{U}(k, t) \mathrm{e}^{i k x_{j}}, 0 \leq j \leq N-1 .
$$

Replacing $F$ and $F^{-1}$ by their discrete counterparts, and discretizing Equations (8) and (9) give

$$
\begin{aligned}
& V\left(x_{j}, t\right)=U\left(x_{j}, t\right)-\left(\frac{2 \pi}{L}\right)^{2} \mu F^{-1}\left\{-k^{2} F(U)\right\}, \\
& \frac{\partial V\left(x_{j}, t\right)}{\partial t}=-3\left(\frac{2 \pi}{L}\right) U^{2}\left(x_{j}, t\right) F^{-1}\{i k F(U)\} .
\end{aligned}
$$

Letting $\boldsymbol{U}=\left[U\left(x_{0}, t\right), U\left(x_{1}, t\right), \cdots, U\left(x_{N-1}, t\right)\right]^{\mathrm{T}}$. Equation (13) can be written in the vector form

$$
\boldsymbol{V}_{t}=\boldsymbol{G}(\boldsymbol{U})
$$

where $\boldsymbol{G}(\boldsymbol{U})$ defines the right hand side of Equation (13).

\section{Fourier Leap-Frog Method for MEW Equation}

A time integration known as a Leap-Frog method (a two-step scheme) is given as

$$
V_{t}=\frac{V(x, t+\Delta t)-V(x, t-\Delta t)}{2 \Delta t}=\frac{V^{n+1}-V^{n-1}}{2 \Delta t}
$$

Use the Leap-Frog scheme to advance in time, we obtain $V(x, t+\Delta t)=V(x, t-\Delta t)+2 \Delta t G(U(t))$.

This is called the Fourier-Leap-Frog (FLF) scheme for the MEW Equation (14). FLF method needs two levels of initial data, we begin with $U(x, 0)$ to get $V(x, 0)$ from Equation (12), we get 


$$
\begin{gathered}
V(x, n \Delta t)=F^{-1}\left((F(U(x, n \Delta t)))\left(1+\left(\frac{2 \pi}{L}\right)^{2} \mu k^{2}\right)\right), \\
V(x, 0)=F^{-1}\left((F(U(x, 0)))\left(1+\left(\frac{2 \pi}{L}\right)^{2} \mu k^{2}\right)\right) .
\end{gathered}
$$

Then evaluate second level of initial solution $V(x, \Delta t)$ by using a higher-order one-step method, for example, a fourth-order Runge-Kutta method (RK4).

$$
\begin{aligned}
& K_{1}=F(U(x, 0), 0) \\
& K_{2}=F\left(U(x, 0)+\frac{1}{2} \Delta t K_{1}, \frac{1}{2} \Delta t\right) \\
& K_{3}=F\left(U(x, 0)+\frac{1}{2} \Delta t K_{2}, \frac{1}{2} \Delta t\right) \\
& K_{4}=F\left(U(x, 0)+\Delta t K_{3}, \Delta t\right) \\
& V(x, \Delta t)=V(x, 0)+\frac{\Delta t}{6}\left[K_{1}+2 K_{2}+2 K_{3}+K_{4}\right]
\end{aligned}
$$

then substitute $V(x, \Delta t)$ in

$$
U(x, n \Delta t)=F^{-1}\left(\frac{F(V(x, n \Delta t))}{1+\left(\frac{2 \pi}{L}\right)^{2} \mu k^{2}}\right)
$$

to get $U(x, \Delta t)$. Thus, the time discretization for Equation (13) is given as

$$
V(x, t+\Delta t)=V(x, t-\Delta t)-2 \Delta t\left(3\left(\frac{2 \pi}{L}\right) U^{2}(x, t) F^{-1}\{i k F(U(x, t))\}\right) .
$$

We substitute $V(x, 0)$ and $U(x, \Delta t)$ in Equation (19) to evaluate $V(x, 2 \Delta t)$ then substitute $V(x, 2 \Delta t)$ in Equation (18) to evaluate $U(x, 2 \Delta t)$, so we have $V(x, \Delta t)$ and $U(x, 2 \Delta t)$, substitute in Equation (19) to evaluate $V(x, 3 \Delta t)$ and evaluate $U(x, 3 \Delta t)$ from Equation (18) and so on, until we evaluate $U(x, t)$ at time $t=n \Delta t$.

\section{Cases Study and Results}

In order to show how good the numerical solutions are in comparison with the exact ones, $L_{2}$ and $L_{\infty}$ error norms will be computed by

$$
\begin{aligned}
& L_{2}=\left\|u^{\text {exact }}-u^{\text {num }}\right\|_{2}=\left[\Delta x \sum_{i=1}^{N}\left|u_{i}^{\text {exact }}-u_{i}^{\text {num }}\right|^{2}\right]^{1 / 2}, \\
& L_{\infty}=\left\|u^{\text {exact }}-u^{\text {num }}\right\|_{\infty}=\max _{i}\left|u_{i}^{\text {exact }}-u_{i}^{\text {num }}\right| .
\end{aligned}
$$

The conservation properties of the MEW equation will be examined by calculating the following three invariants, given as [17] which respectively correspond to mass, momentum, and energy

$$
\begin{aligned}
& C_{1}=\int_{a}^{b} U \mathrm{~d} x=\Delta x \sum_{j=1}^{n} U\left(x_{j}, t\right), \\
& C_{2}=\int_{a}^{b}\left[U^{2}+\mu\left(U_{x}\right)^{2}\right] \mathrm{d} x=\Delta x \sum_{j=1}^{n}\left[\left(U\left(x_{j}, t\right)\right)^{2}+\mu\left(U_{x}\left(x_{j}, t\right)\right)^{2}\right], \\
& C_{3}=\int_{a}^{b} U^{4} \mathrm{~d} x=\Delta x \sum_{j=1}^{n}\left(U\left(x_{j}, t\right)\right)^{4} .
\end{aligned}
$$


For the computation of $U_{x}$ in Equation (21), we used Fourier transform. To implement the performance of the method, three test problems will be considered: the motion of a single solitary wave, development of two positive solitary waves interaction, development of three positive solitary wave interaction.

\subsection{The Motion of Single Solitary Wave}

Consider Equation (1) with the boundary $U \rightarrow 0$ as $x \rightarrow \pm \infty$ and initial condition

$$
U(x, 0)=A \operatorname{sech}\left[k\left(x-x_{0}\right)\right] \text {. }
$$

This problem has a solitary wave solution of the form

$$
U(x, t)=A \sec h\left[k\left(x-x_{0}-v t\right)\right]
$$

which represents the motion of a single solitary wave with amplitude $A$, where the wave velocity $v=A^{2} / 2$ and $k=\sqrt{1 / \mu}$. For this problem the analytical values of the invariants are [16]

$$
C_{1}=\frac{A \pi}{k}, C_{2}=\frac{2 A^{2}}{k}+\frac{2 \mu k A^{2}}{3}, C_{3}=\frac{4 A^{4}}{3 k} .
$$

For the numerical simulation of the motion of a single solitary wave, the parameters $\Delta x=0.1, \Delta t=0.001, \mu=$ $1, x_{0}=30, N=2048$ and $A=0.25$ are chosen. The analytical values for the invariants are $C_{1}=0.7853982, C_{2}=$ 0.1666667 , and $C_{3}=0.0052083$. As it is seen from Table 1 , the invariants $C_{1}$ and $C_{3}$ remain almost constant during the computer run at times $t=0$ to $t=100$ (changes of the invariants $C_{1}$ and $C_{3}$ approach zero), where $C_{2}$ changes from its initial value by less than $1 \times 10^{-9}$. The error norms $L_{2}$ and $L_{\infty}$ at different various times are shown in Table 1. It is shown that the numerical values very close to the exact values. Figure 1(a) shows that the proposed method performs the motion of propagation of a solitary wave satisfactorily, which moved to the right at a constant speed and preserved its amplitude and shape with increasing time as expected. Amplitude is 0.25 at $t=0$ which is located at $x=30$, while it is 0.249985 at $t=20$ which is located at $x=30.6149$. The absolute difference in amplitudes at times $t=0$ and $t=20$ is only $1.5 \times 10^{-5}$. Error distribution at time $t=20$ is drawn in Figure 1(b), from which it can be seen that maximum errors happened just around the peak position of the solitary wave. Table 2 displays the values of the error norms and numerical invariants obtained at different values of $N$ with $\Delta x=0.1, \Delta t=0.001, \mu=1, x_{0}=30$ and $A=0.25$. As it is seen from Table 2, the error norms decrease (halved) when $N$ increases (doubled) and numerical invariants $C_{1}, C_{2}$ and $C_{3}$ closed to the analytical values when $N$ increases. The comparison between the results obtained by the present with those in the other studies [15] [22] [24] [25] also documented in Table 2.

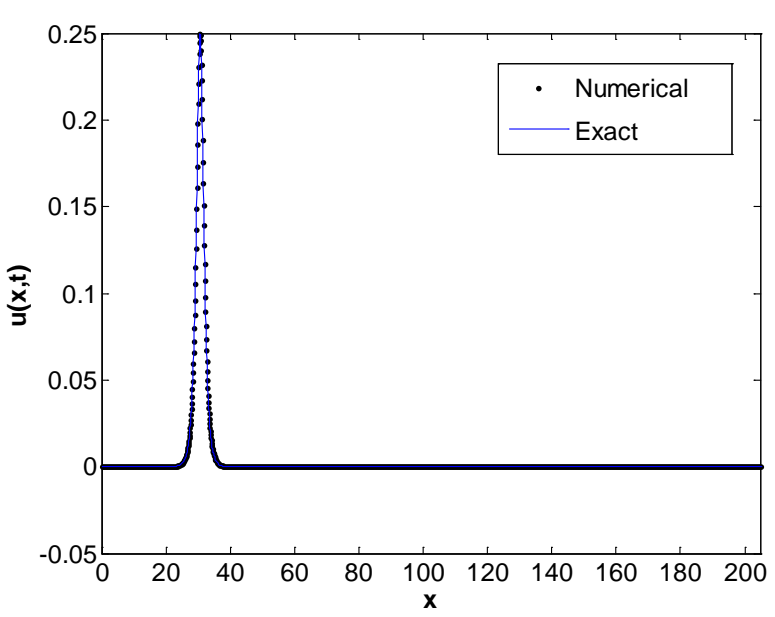

(a)

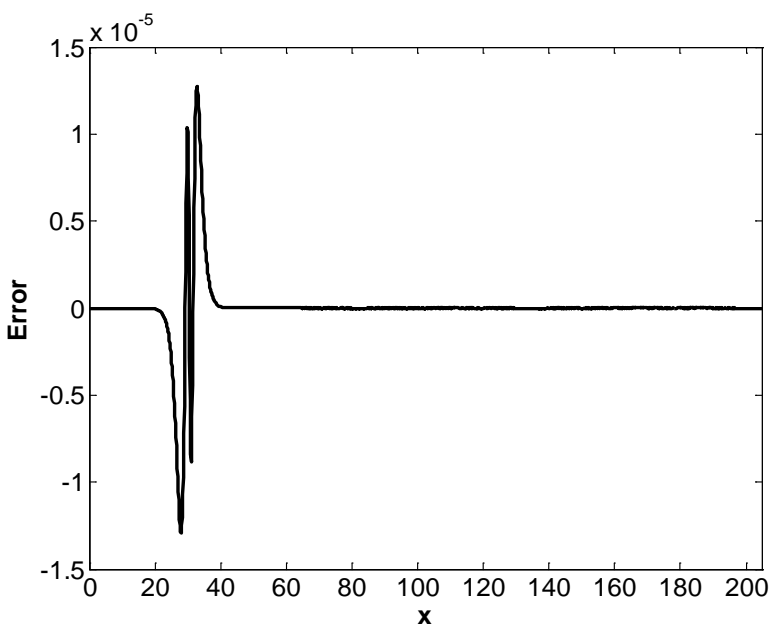

(b)

Figure 1. (a) The motion of a single solitary wave and (b) the error distribution in FLF scheme for MEW equation with $A=$ $0.25, N=2048, \Delta x=0.1$ and $\Delta t=0.001$ at $t=20$. 
Table 1. Invariants and error norms for the single soliton using FLF scheme with $A=0.25, N=2048, \Delta x=0.1$ and $\Delta t=$ 0.001 .

\begin{tabular}{cccccc}
\hline$t$ & $C_{1}$ & $C_{2}$ & $C_{3}$ & $L_{2} \times 10^{3}$ & $L_{\infty} \times 10^{3}$ \\
\hline 0 & 0.785014668 & 0.166625987 & 0.005205790 & 0.0000000 & 0.0000000 \\
5 & 0.785014668 & 0.166625987 & 0.005205790 & 0.0069317 & 0.0032278 \\
10 & 0.785014668 & 0.166625986 & 0.005205790 & 0.0160855 & 0.0081248 \\
15 & 0.785014668 & 0.166625986 & 0.005205790 & 0.0226029 & 0.0113299 \\
20 & 0.785014668 & 0.166625986 & 0.005205790 & 0.0275859 & 0.0129414 \\
25 & 0.785014668 & 0.166625986 & 0.005205790 & 0.0343768 & 0.0161599 \\
30 & 0.785014668 & 0.166625986 & 0.005205790 & 0.0410980 & 0.0193344 \\
35 & 0.785014668 & 0.166625986 & 0.005205790 & 0.0477366 & 0.0224764 \\
40 & 0.785014668 & 0.166625986 & 0.005205790 & 0.0558158 & 0.0273561 \\
45 & 0.785014668 & 0.166625986 & 0.005205790 & 0.0622846 & 0.0305792 \\
50 & 0.785014668 & 0.166625986 & 0.005205790 & 0.0686557 & 0.0337939 \\
100 & 0.785014668 & 0.166625986 & 0.005205790 & 0.1239589 & 0.0654669
\end{tabular}

Table 2. Invariants, error norms for the single soliton MEW equation using FLF scheme with $A=0.25, \Delta x=0.1$ and $\Delta t=$ 0.001 at different values of $N$ at $t=20$ and comparison with different methods at $A=0.25, \Delta t=0.05$ and $\Delta x=0.1$.

\begin{tabular}{|c|c|c|c|c|c|}
\hline$N$ & $C_{1}$ & $C_{2}$ & $C_{3}$ & $L_{2} \times 10^{3}$ & $L_{\infty} \times 10^{3}$ \\
\hline 512 & 0.7838642 & 0.1665041 & 0.0051982 & 0.1103535 & 0.0517942 \\
\hline 1024 & 0.7846312 & 0.1665853 & 0.0052033 & 0.0551734 & 0.0258918 \\
\hline 2048 & 0.7850147 & 0.1666260 & 0.0052058 & 0.0275859 & 0.0129412 \\
\hline 4096 & 0.7852064 & 0.1666463 & 0.0052071 & 0.0137927 & 0.0064692 \\
\hline 8192 & 0.7853023 & 0.1666565 & 0.0052077 & 0.0068963 & 0.0032342 \\
\hline Ref [15] & 0.7853898 & 0.1667614 & 0.0052082 & 0.0796940 & 0.0465523 \\
\hline Ref [22] & 0.7849545 & 0.1664765 & 0.0051995 & 0.2905166 & 0.2498925 \\
\hline Ref [24] & 0.7853977 & 0.1664735 & 0.0052083 & 0.2692812 & 0.2569972 \\
\hline Ref [25] & 0.7853967 & 0.1666663 & 0.0052083 & 0.0800980 & 0.0460618 \\
\hline
\end{tabular}

\subsection{Interaction of Two Solitary Waves}

The initial condition given by the linear sum of two separate solitary waves of various amplitudes

$$
u(x, 0)=\sum_{j=1}^{2} A_{j} \operatorname{sech}\left(k\left(x-x_{j}\right)\right),
$$

where $k=1 / \sqrt{\mu}$. Firstly the interaction of two positive solitary waves is study with the parameters $A_{1}=1, A_{2}=$ $0.5, x_{1}=15, x_{2}=30, N=8192, \Delta x=0.1$ and $\Delta t=0.01$. The analytic invariants are [25], $C_{1}=\pi\left(A_{1}+A_{2}\right)=4.7123889, C_{2}=(8 / 3)\left(A_{1}^{2}+A_{2}^{2}\right)=3.3333333$ and $C_{3}=(4 / 3)\left(A_{1}^{4}+A_{2}^{4}\right)=1.4166667$. The initial function was placed on the left side of the region with the larger wave to the left of the smaller one as seen in Figure 2(a). Both waves move to the right with velocities dependent upon their magnitudes. The larger wave catches up with the smaller one as time increase. Interaction started at about time $t=25$, the overlapping process continues until the time $t=40$, then two solitary waves emerge from the interaction and resume their former shapes and amplitudes as shown in Figures 2(b)-(f). The magnitude of the smaller wave 0.510741 on reaching 


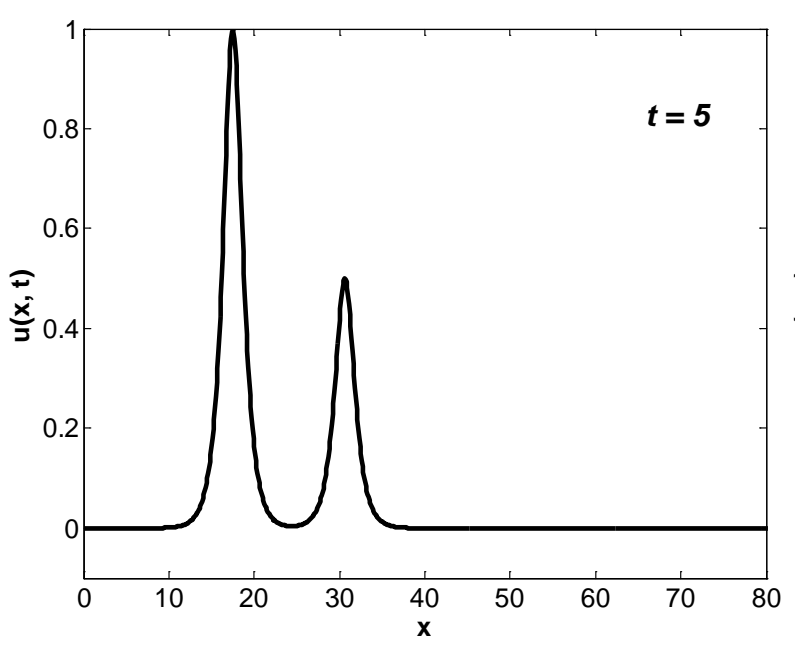

(a)

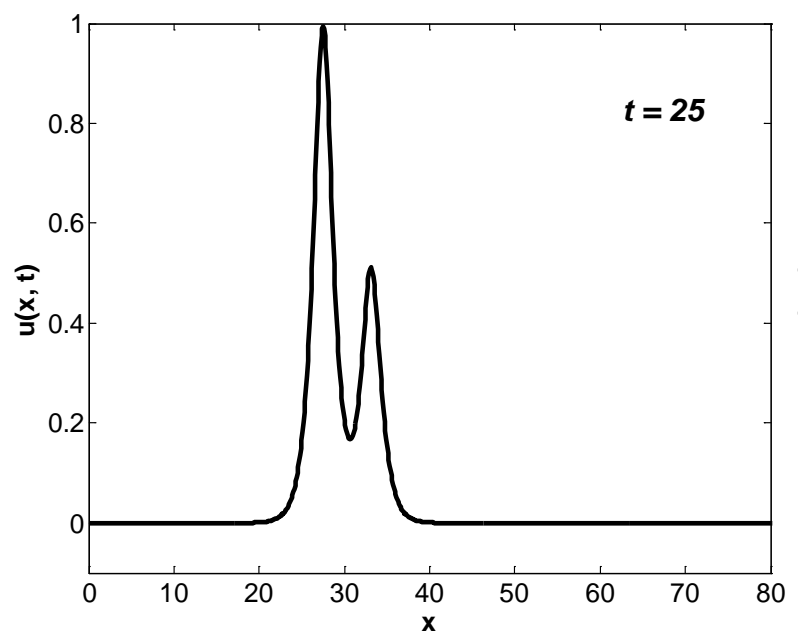

(c)

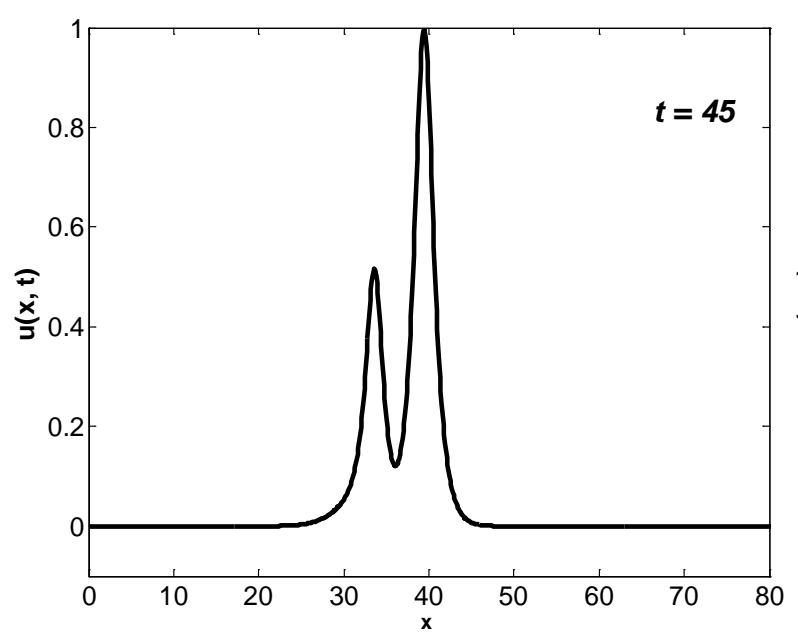

(e)

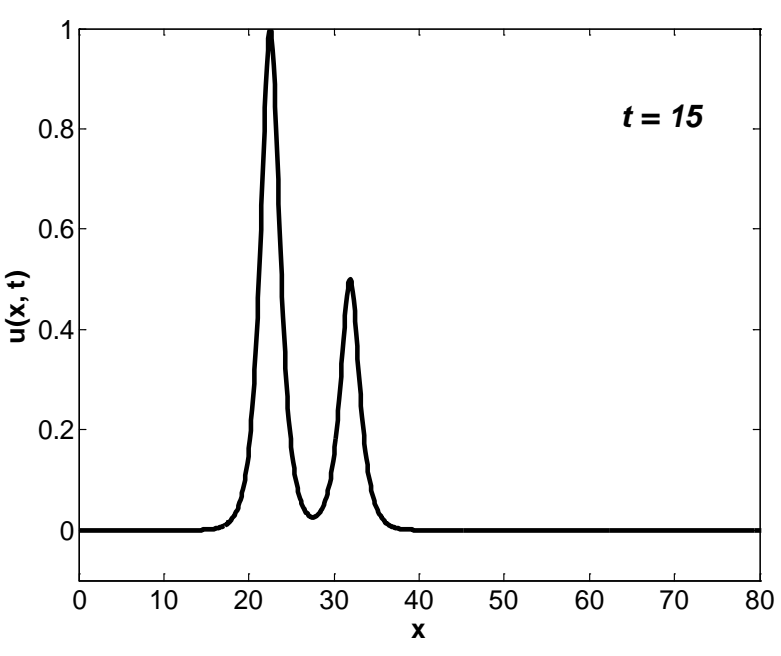

(b)

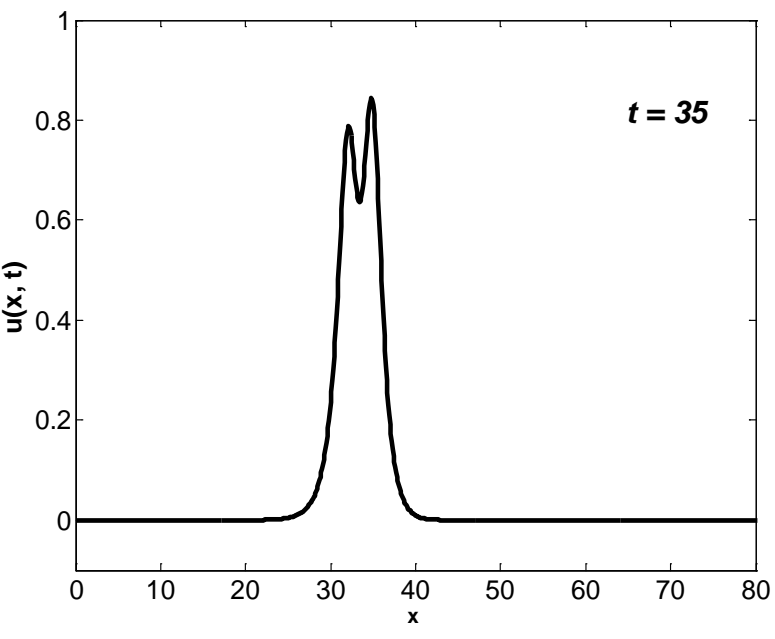

(d)

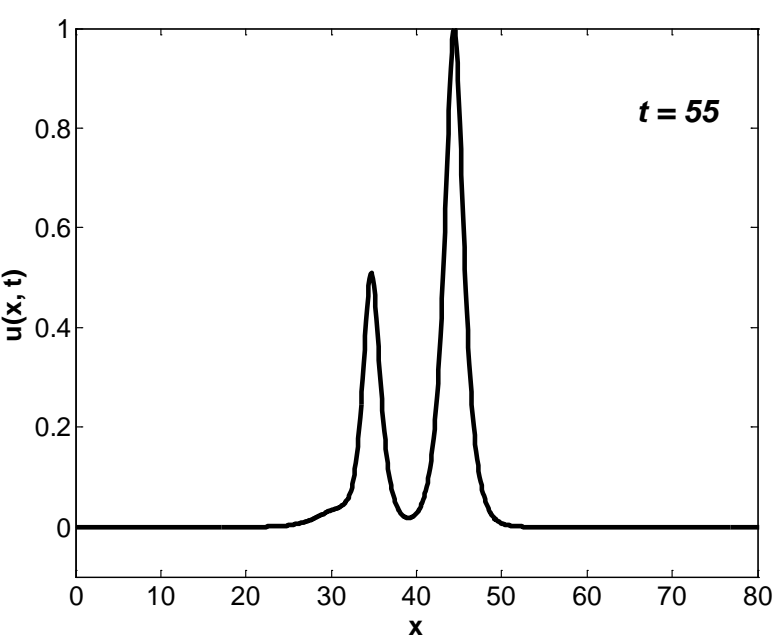

(f)

Figure 2. Interaction of two solitary waves at different times with $A_{1}=1$ and $A_{2}=0.5$. 
position $x=34.7$ and of the larger wave 1.000097 having the position $x=44.4$ are measured at time $t=55$ so that difference in amplitudes is 0.010741 for the smaller wave and 0.000097 for the larger wave. Table 3 displays the values of the invariants obtained by the present method. It is observed that the obtained values of the invariants remain almost constant during the computer run. The change in $C_{2}$ is $6.11 \times 10^{-5}$ and in $C_{3}$ is $5.68 \times$ $10^{-5}$ and $C_{1}$ is exact up to the last recorded digit.

The intersection of two solitary waves was also studies with the following parameters: $\mu=1, x_{1}=15, x_{2}=30$, $A_{1}=-2, A_{2}=1, N=8192, \Delta t=0.01$ and $\Delta x=0.1$ in the range $0 \leq x \leq 819.2$. The experiment was run from $t=0$ to $t=55$ to allow the interaction to take place. Figure 3 shows the development of the solitary wave interaction. As is seen from Figure 3, at $t=0$ a wave with the negative amplitude is on the left of another wave with the positive amplitude. The larger wave with the negative amplitude catches up with the smaller one with the positive amplitude as the time increases. At $t=55$, the amplitude of the smaller wave is at the point 0.9741792 at the point 52.5064095 whereas the amplitude of the larger one is -2.0014682 at the point 123.6150897326334 It is found that the absolute difference in amplitudes is 0.025820781 for the smaller wave and 0.00146821 for the larger wave. The analytical invariants can be found as $C_{1}=-3.1415927, C_{2}=13.3333333$ and $C_{2}=22.6666667$. It can be seen in Table 3 that the values obtained for the invariants are satisfactorily constant during the computer run.

\subsection{Interaction of Three Solitary Waves}

Interaction of three solitary waves is studied by considering Equation (1) with the following initial condition:

$$
u(x, 0)=\sum_{j=1}^{3} A_{j} \operatorname{sech}\left(k\left(x-x_{j}\right)\right),
$$

where $=1 / \sqrt{\mu}$. The computations are carried out with parameters $=1, A_{1}=1, A_{2}=0.5, A_{3}=0.25, x_{1}=15, x_{2}=$ 30, $x_{3}=45, N=8192, \Delta x=0.1$ and $\Delta t=0.01$. Solitary wave having the largest amplitude is located to the left of the smaller ones. As is well known, solitary waves with larger amplitudes have a greater velocity than those with smaller amplitudes. Consequently, as time goes on the larger two solitary waves catches up with the smaller one, the overlapping process of the three solitary waves continues while the larger solitary waves have overtaken the smaller ones. Plot of the three solitary waves is depicted at various times in Figure 4. Interaction of three solitary

Table 3. Invariants for the interaction of two solitary waves with $\Delta t=0.01, \Delta x=0.1$ and $N=8192$.

\begin{tabular}{ccccccc}
\hline$t$ & \multicolumn{3}{c}{$A_{1}=1, A_{2}=0.5$} & & $A_{1}=-2, A_{2}=1$ \\
\cline { 2 - 6 } & $C_{1}$ & $C_{2}$ & $C_{3}$ & $C_{1}$ & $C_{2}$ & $C_{3}$ \\
\hline 5 & 4.7118132 & 3.3331323 & 1.4164968 & -3.1412080 & 13.3325098 & 22.6638508 \\
10 & 4.7118132 & 3.3330876 & 1.4164524 & -3.1412080 & 13.3293775 & 22.6514303 \\
15 & 4.7118132 & 3.3330830 & 1.4164479 & -3.1412080 & 13.3306037 & 22.6526691 \\
20 & 4.7118132 & 3.3330828 & 1.4164480 & -3.1412080 & 13.3293817 & 22.6514527 \\
25 & 4.7118132 & 3.3330828 & 1.4164480 & -3.1412080 & 13.3293781 & 22.6514501 \\
30 & 4.7118132 & 3.3330822 & 1.4164476 & -3.1412080 & 13.3293776 & 22.6514500 \\
35 & 4.7118132 & 3.3330776 & 1.4164476 & -3.1412080 & 13.3293774 & 22.6514500 \\
40 & 4.7118132 & 3.3330712 & 1.4164400 & -3.1412080 & 13.3293774 & 22.6514500 \\
45 & 4.7118132 & 3.3330803 & 1.4164462 & -3.1412080 & 13.3293773 & 22.6514500 \\
50 & 4.7118132 & 3.3330826 & 1.4164478 & -3.1412080 & 13.3293749 & 22.6514500 \\
\hline 55 & 4.7118132 & 3.3330831 & 1.4164481 & -3.1412080 & 13.3293750 & 22.6514500 \\
\hline
\end{tabular}




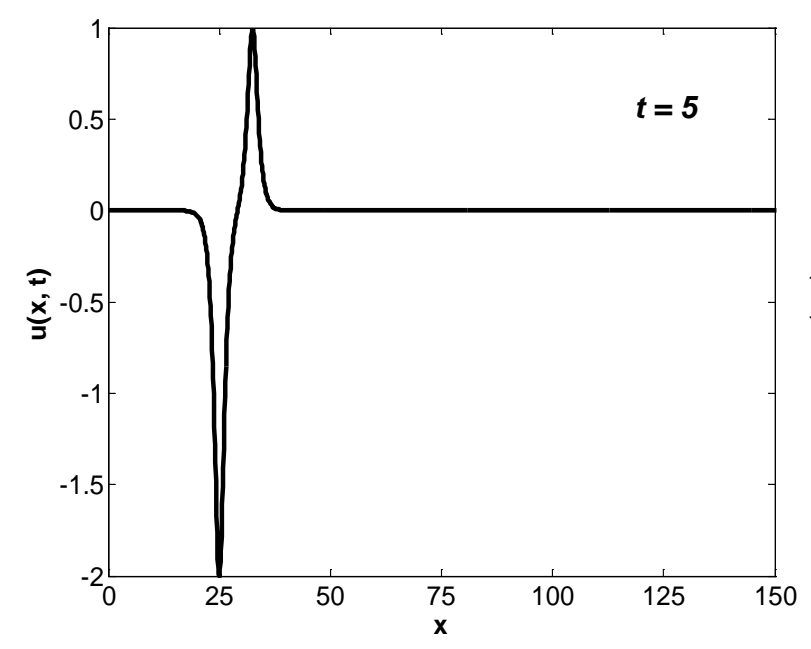

(a)

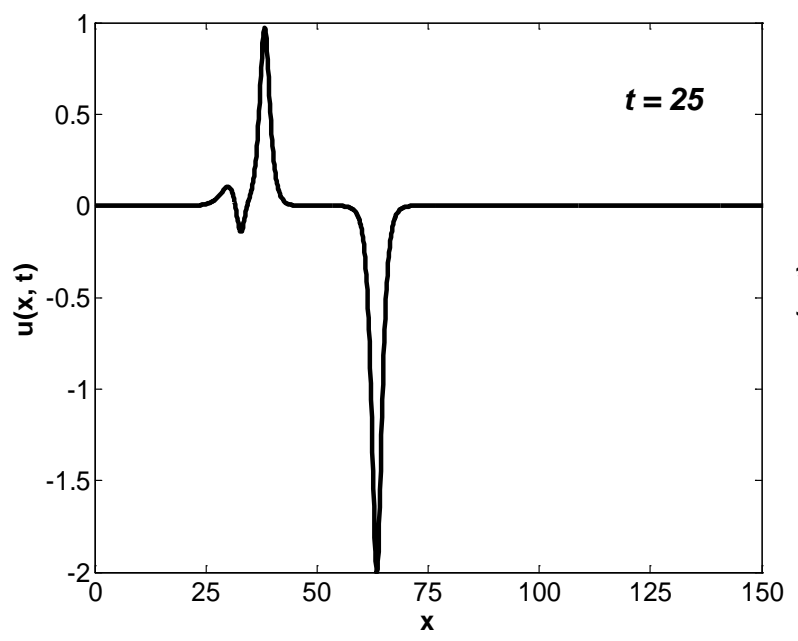

(c)

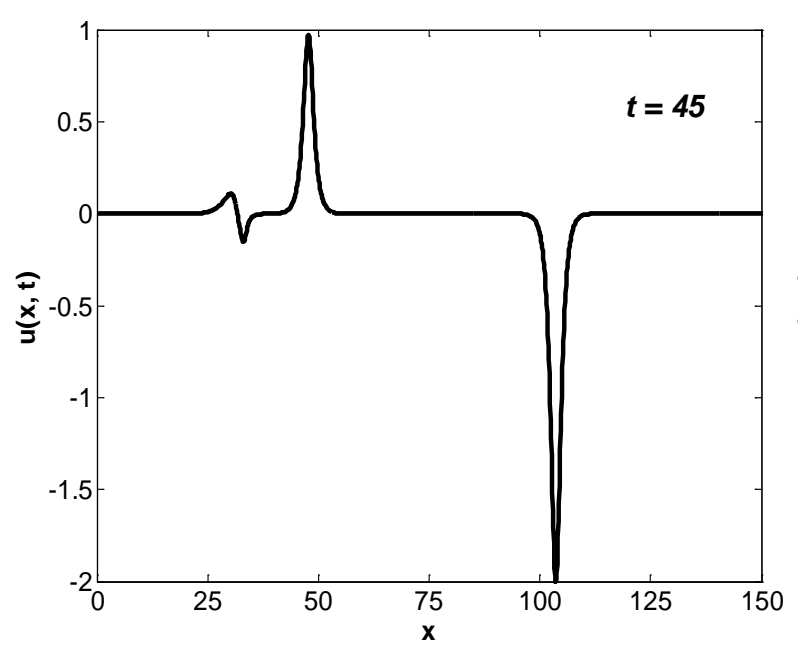

(e)

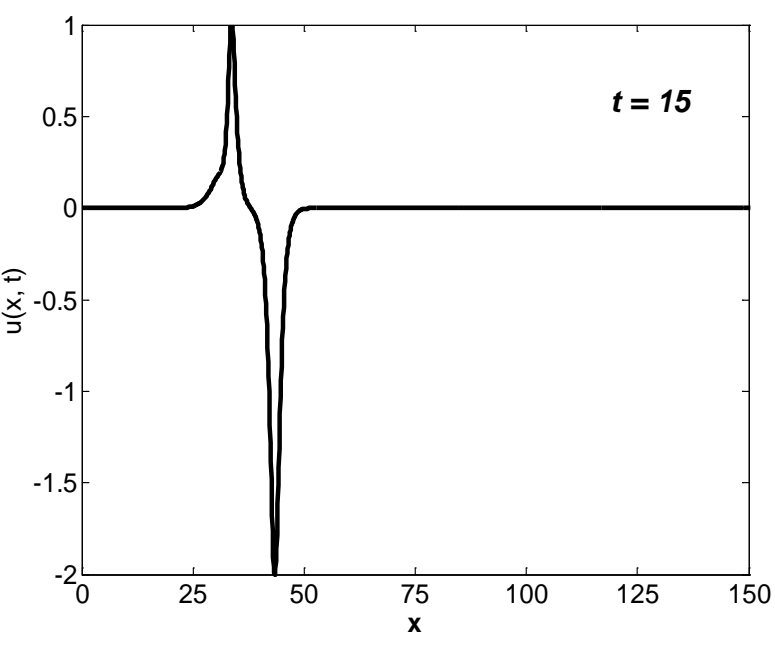

(b)

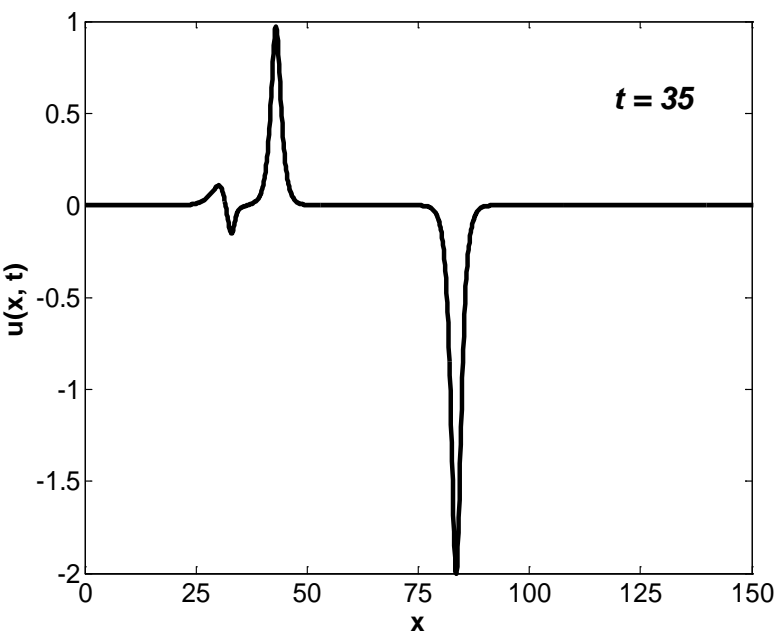

(d)

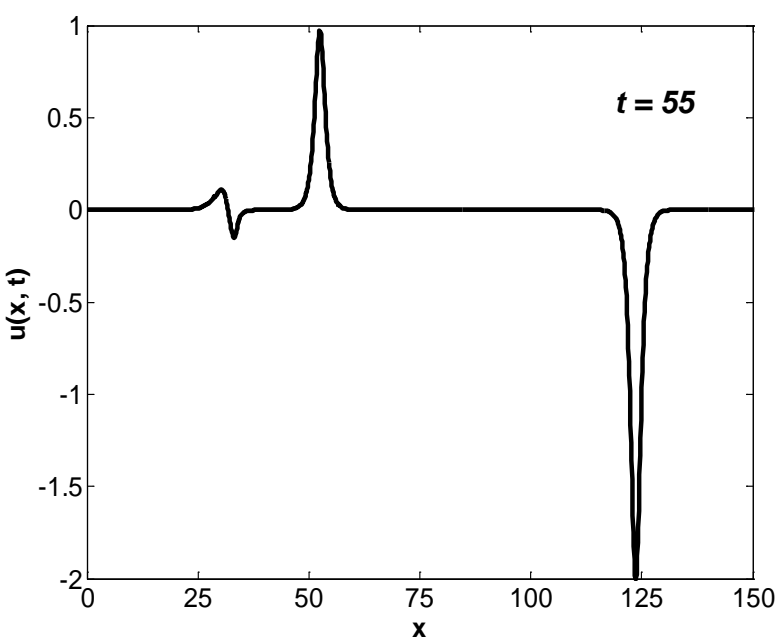

(f)

Figure 3. Interaction of two solitary waves at different times with $A_{1}=-2$ and $A_{2}=1$. 
H. N. Hassan

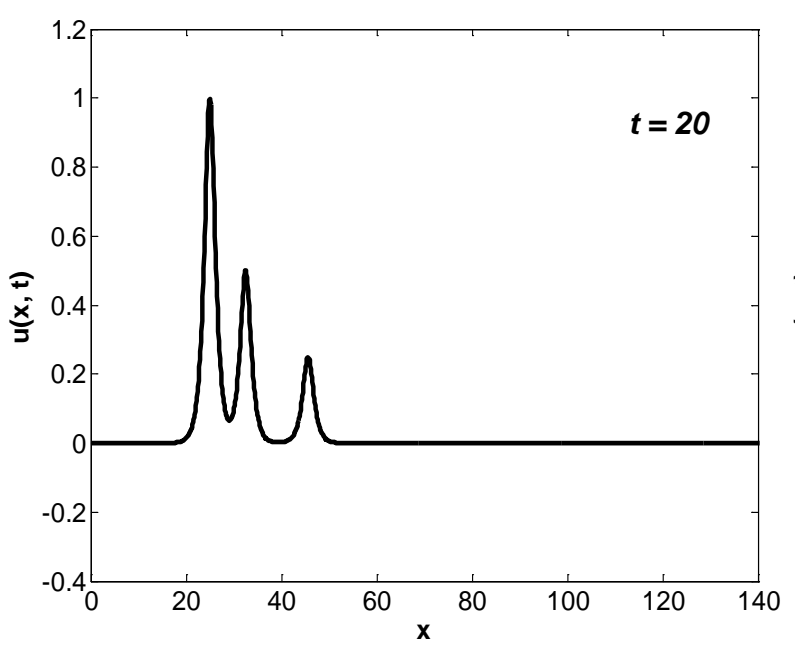

(a)

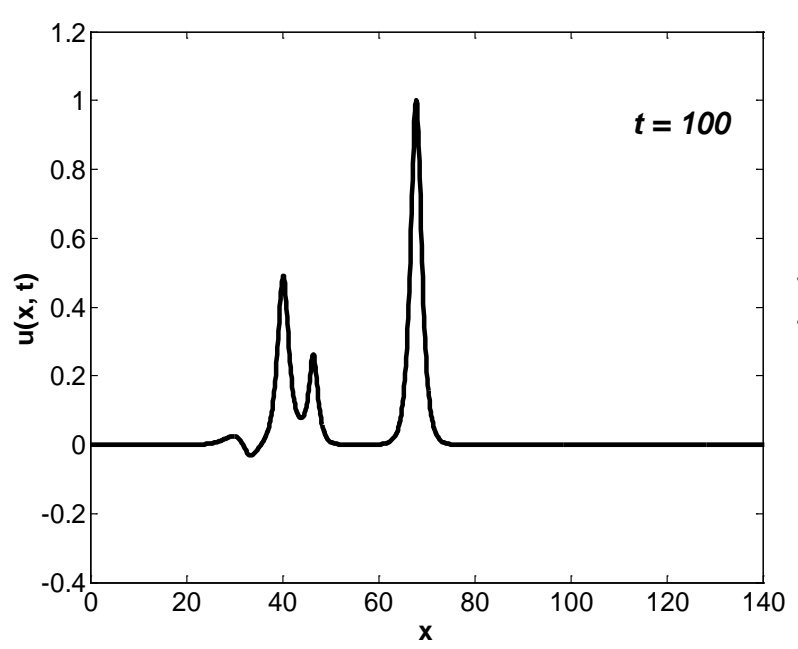

(c)

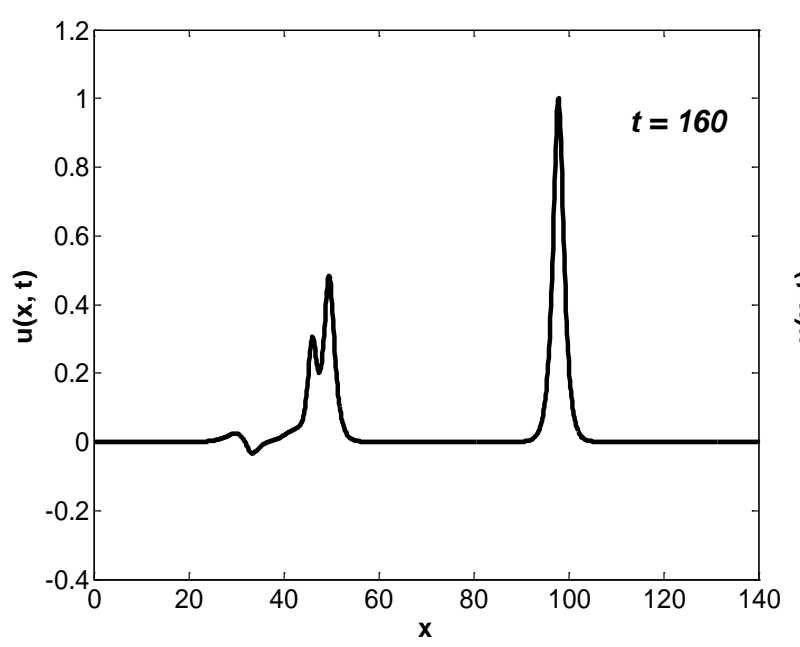

(e)

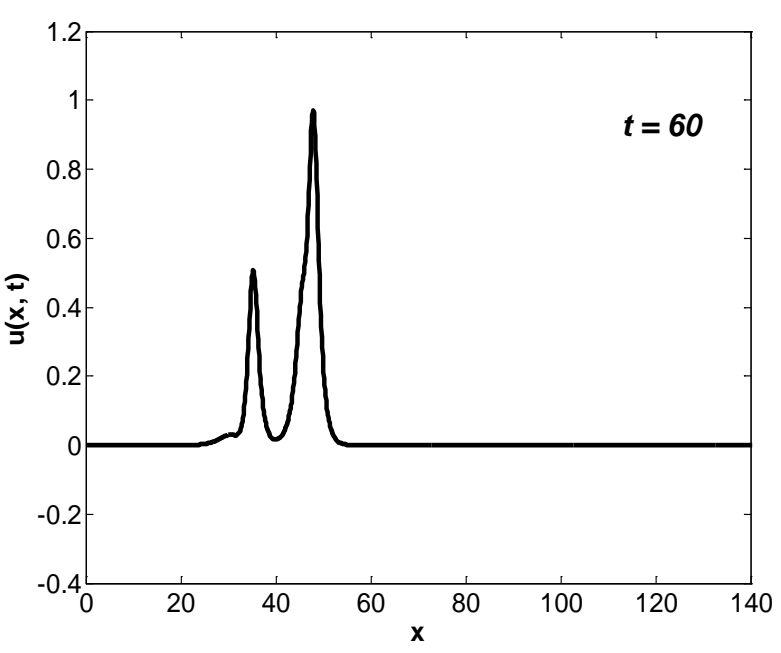

(b)

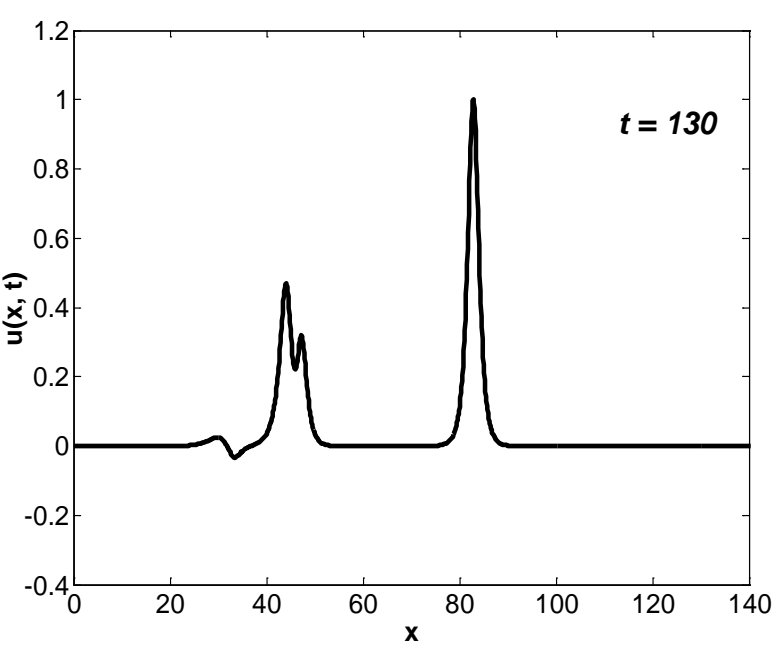

(d)

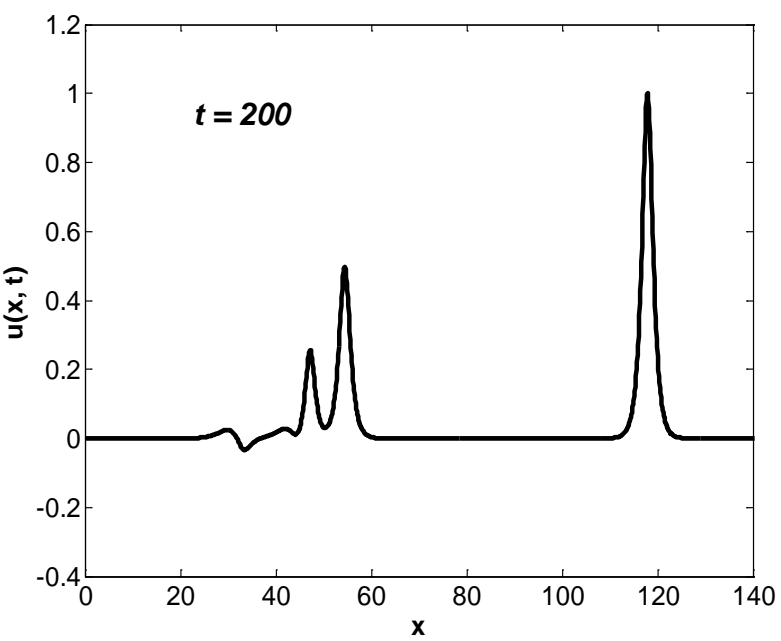

(f)

Figure 4. Interaction of three solitary waves at different times. 
waves can be openly observed from the time-amplitude graph in Figure 4 for the three algorithms. At $t=200$, the amplitudes of the smaller waves are 0.25613 at the point $x=47.21$ and 0.49672 at the point $x=54.41$, whereas the amplitude of the larger one is 1.00032 at the point $x=117.91$. Table 4 displays the values of the invariants obtained by the present method. It is observed that the obtained values of the invariants remain almost constant during the computer run. The change in $C_{2}$ is $5.37 \times 10^{-5}$ and in $C_{3}$ is $5.09 \times 10^{-5}$ and $C_{1}$ is exact up to the last recorded digit. The analytical values can be found [25] as $C_{1}=\pi\left(A_{1}+A_{2}+A_{3}\right)=5.4977871$, $C_{2}=(8 / 3)\left(A_{1}^{2}+A_{2}^{2}+A_{3}^{2}\right)=3.5$ and $C_{3}=(4 / 3)\left(A_{1}^{4}+A_{2}^{4}+A_{3}^{4}\right)=1.421875$.

\subsection{The Maxwellian Initial Condition}

We consider here is the numerical solution of the Equation (1) with the Maxwellian initial condition

$$
u(x, 0)=\mathrm{e}^{-x^{2}},
$$

with the boundary conditions

$$
u(-20, t)=u_{x}(-20, t)=u(20, t)=u_{x}(20, t)=0 .
$$

As it is known, Maxwellian initial condition the behavior of the solution depends on the values of $\mu$. The computations are carried out for the cases $\mu=1,0.5,0.1,0.05,0.02$ and 0.005 which are used in [12] [17] [21]. When $\mu=1,0.5$ is used as shown Figure 5(a) and Figure 5(b) at time $t=12$ the Maxwellian initial condition does not cause development into a clean solitary wave. However with smaller values of $\mu=1,0.1,0.05,0.02$ and 0.005 Maxwellian initial condition breaks up into more solitary waves which drawn in Figures 5(c)-(f) at time $t=12$. The numerical conserved quantities with $\mu=1,0.5,0.1,0.05,0.02$ and 0.005 are given in Table 5 . It can be seen in Table 4 that the values obtained for the invariants are satisfactorily constant during the computer run.

\section{Conclusion}

The Fourier Leap Frog method has been successfully applied to obtain the numerical solution of the modified equal width wave equation. Four test problems are worked out to examine the performance of the used method. The motion of a single solitary wave and its accuracy was shown by calculating error norms $L_{2}$ and $L_{\infty}$ and shown in the figures and tables. The interaction of two solitary waves and its accuracy shown by compare with other numerical solutions. The interaction of three solitary waves and its accuracy shown by compare with other numerical solutions. A Maxwellian initial condition pulse is then studied at different values of $\mu$. The invariants

Table 4. Invariants for the interaction of three solitary waves.

\begin{tabular}{cccc}
\hline$t$ & $C_{1}$ & $C_{2}$ & $C_{3}$ \\
\hline 0 & 5.4971155 & 3.4997894 & 1.4217047 \\
20 & 5.4971155 & 3.4997399 & 1.4216558 \\
40 & 5.4971155 & 3.4997374 & 1.4216540 \\
60 & 5.4971155 & 3.4997357 & 1.4216538 \\
80 & 5.4971155 & 3.4997401 & 1.4216560 \\
100 & 5.4971155 & 3.4997401 & 1.4216560 \\
120 & 5.4971155 & 3.4997401 & 1.4216560 \\
140 & 5.4971155 & 3.4997400 & 1.4216559 \\
160 & 5.4971155 & 3.4997401 & 1.4216560 \\
180 & 5.4971155 & 3.4997401 & 1.4216560 \\
200 & 5.4971155 & 3.4997401 & 1.4216560 \\
\hline
\end{tabular}




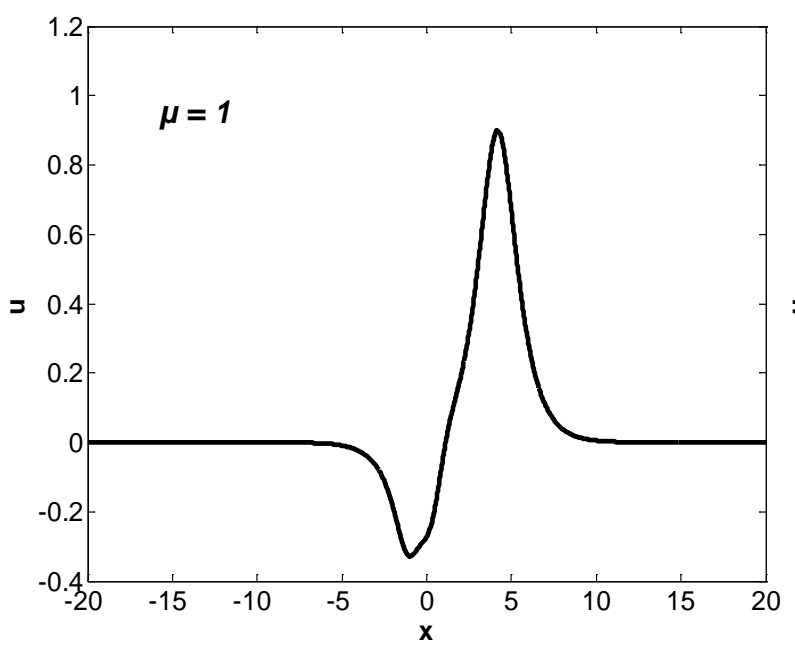

(a)

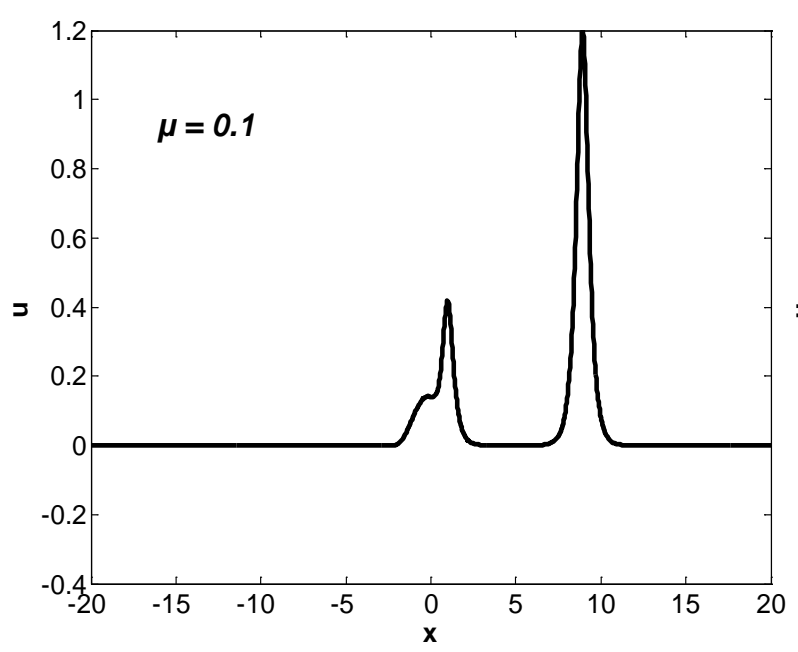

(c)

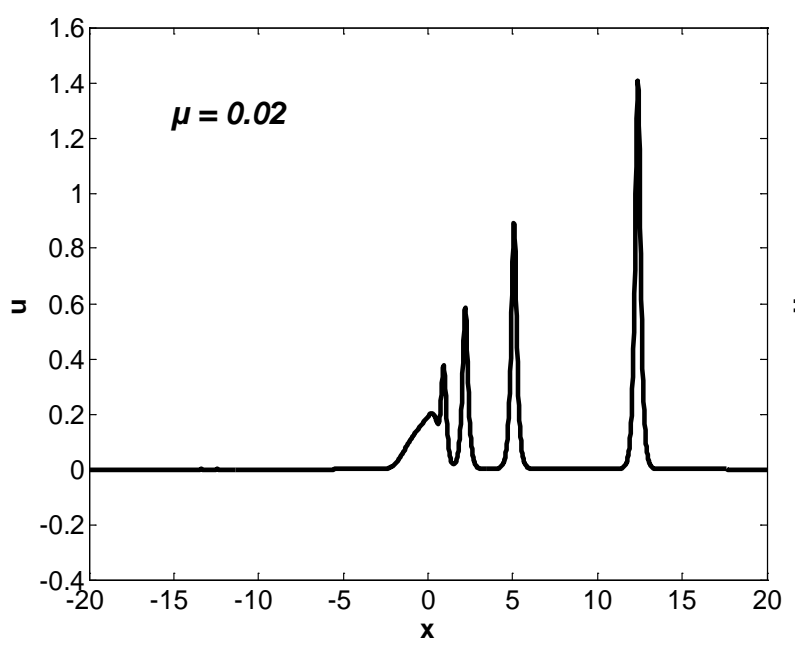

(e)

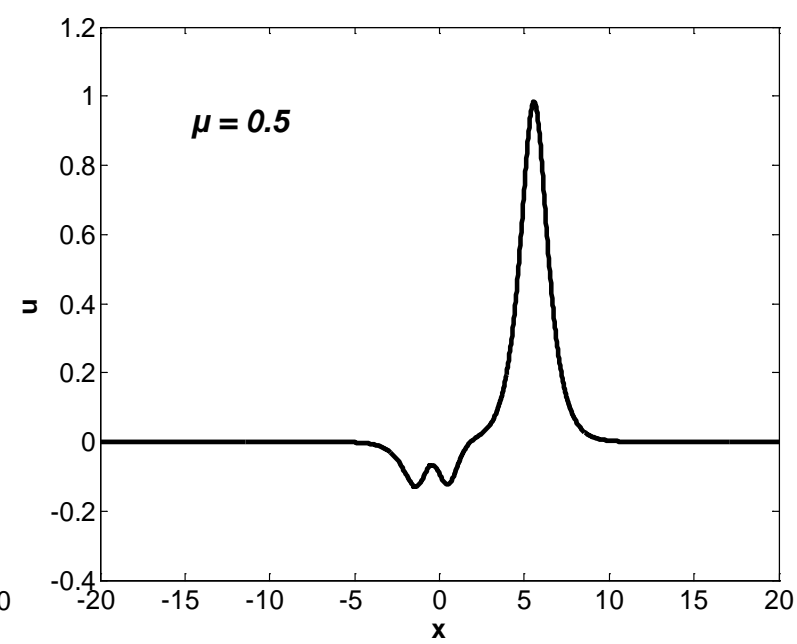

(b)

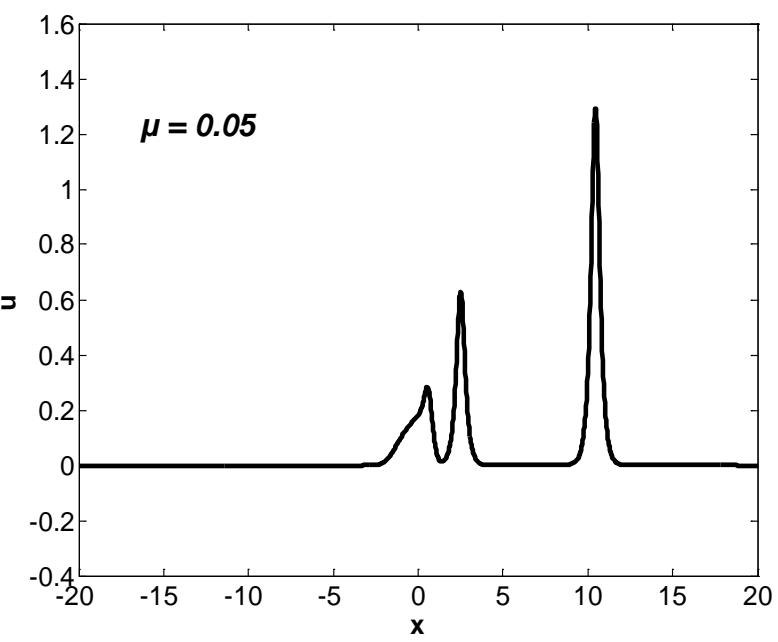

(d)

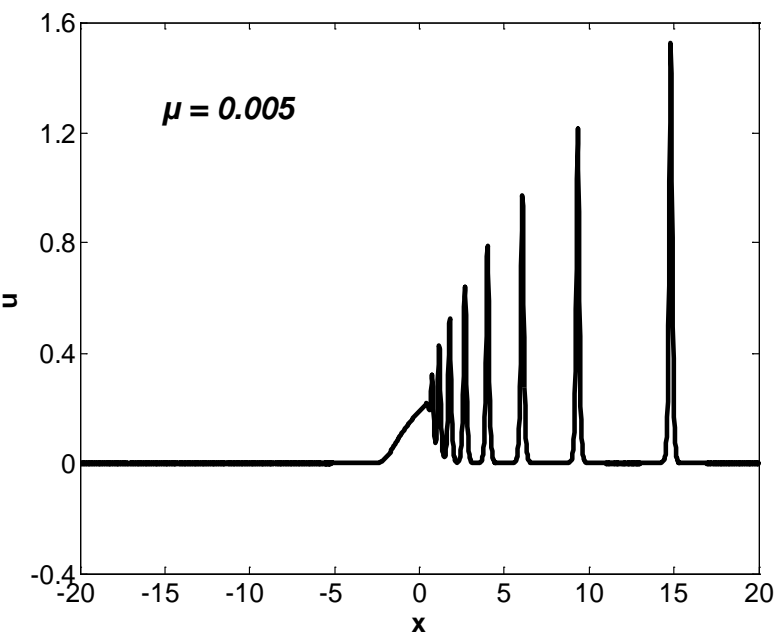

(f)

Figure 5. Maxwellian initial condition, state at $t=12$ and different values of $\mu$. 
Table 5. Invariants for Maxwellian initial condition at different values of $\mu$.

\begin{tabular}{|c|c|c|c|c|}
\hline$t$ & $\mu$ & $C_{1}$ & $C_{2}$ & $C_{3}$ \\
\hline 0 & & 1.7715884 & 2.5066286 & 0.8857942 \\
\hline 3 & & 1.7715884 & 2.5066279 & 0.8857938 \\
\hline 6 & 1 & 1.7715884 & 2.5066276 & 0.8857936 \\
\hline 9 & & 1.7715884 & 2.5066277 & 0.8857937 \\
\hline 12 & & 1.7715884 & 2.5066275 & 0.8857937 \\
\hline 0 & & 1.7715884 & 1.8796654 & 0.8857942 \\
\hline 3 & & 1.7715884 & 1.8796645 & 0.8857934 \\
\hline 6 & 0.5 & 1.7715884 & 1.8796646 & 0.8857934 \\
\hline 9 & & 1.7715884 & 1.8796646 & 0.8857935 \\
\hline 12 & & 1.7715884 & 1.8796645 & 0.8857935 \\
\hline 0 & & 1.7715884 & 1.3780948 & 0.8857942 \\
\hline 3 & & 1.7715884 & 1.3780956 & 0.8857942 \\
\hline 6 & 0.1 & 1.7715884 & 1.3780957 & 0.8857942 \\
\hline 9 & & 1.7715884 & 1.3780955 & 0.8857941 \\
\hline 12 & & 1.7715884 & 1.3780955 & 0.8857941 \\
\hline 0 & & 1.7715884 & 1.3153985 & 0.8857942 \\
\hline 3 & & 1.7715884 & 1.3154016 & 0.8857967 \\
\hline 6 & 0.05 & 1.7715884 & 1.3154017 & 0.8857967 \\
\hline 9 & & 1.7715884 & 1.3154017 & 0.8857967 \\
\hline 12 & & 1.7715884 & 1.3154016 & 0.8857967 \\
\hline 0 & & 1.7715884 & 1.2777807 & 0.8857942 \\
\hline 3 & & 1.7715884 & 1.2777913 & 0.8858065 \\
\hline 6 & 0.02 & 1.7715884 & 1.2777914 & 0.8858066 \\
\hline 9 & & 1.7715884 & 1.2777913 & 0.8858066 \\
\hline 12 & & 1.7715884 & 1.2777914 & 0.8858066 \\
\hline 0 & & 1.7715884 & 1.2589718 & 0.8857942 \\
\hline 3 & & 1.7715884 & 1.2590204 & 0.8858617 \\
\hline 6 & 0.005 & 1.7715884 & 1.2590208 & 0.8858619 \\
\hline 9 & & 1.7715884 & 1.2590209 & 0.8858619 \\
\hline 12 & & 1.7715884 & 1.2590209 & 0.8858619 \\
\hline
\end{tabular}

are satisfactorily constant in computer run in all cases. The obtained results show that the present method is a remarkably successful numerical method and can also be efficiently applied to other types of non-linear problems.

\section{References}

[1] Fornberg, B. (1996) A Practical Guide to Pseudospectral Methods. Cambridge University Press, New York. http://dx.doi.org/10.1017/CBO9780511626357 
[2] Fornberg, B. and Whitham, G.B. (1978) A Numerical and Theoretical Study of Certain Nonlinear Wave Phenomena. Philosophical Transactions of the Royal Society of London, 289, 373-404. http://dx.doi.org/10.1098/rsta.1978.0064

[3] Hassan, H.N. and Saleh, H.S. (2013) Fourier Spectral Methods for Solving Some Nonlinear Partial Differential Equations. International Journal of Open Problems in Computer Science and Mathematics, 6, 144-179. http://dx.doi.org/10.12816/0006177

[4] Hassan, H.N. (2010) Numerical Solution of a Boussinesq Type Equation Using Fourier Spectral Methods. Zeitschrift für Naturforschung A, 65, 305-314. http://dx.doi.org/10.1515/zna-2010-0407

[5] Borluk, H. and Muslu, G.M. (2015) A Fourier Pseudospectral Method for a Generalized Improved Boussinesq Equation. Numerical Methods for Partial Differential Equations, 31, 995-1008. http://dx.doi.org/10.1002/num.21928

[6] Hassan, H.N. and Saleh, H.S. (2010) The Solution of the Regularized Long Wave Equation Using the Fourier LeapFrog Method. Zeitschrift für Naturforschung A, 65, 268-276. http://dx.doi.org/10.1515/zna-2010-0402

[7] Gardner, L.R.T. and Gardner, G.A. (1990) Solitary Waves of the Regularized Long-Wave Equation. Journal of Computational Physics, 91, 441-459. http://dx.doi.org/10.1016/0021-9991(90)90047-5

[8] Gardner, L.R.T. and Gardner, G.A. (1992) Solitary Waves of the Equal Width Wave Equation. Journal of Computational Physics, 101, 218-223. http://dx.doi.org/10.1016/0021-9991(92)90054-3

[9] Morrison, P.J., Meiss, J.D. and Cary, J.R. (1984) Scattering of Regularized-Long-Wave Solitary Waves. Physica D. Nonlinear Phenomena, 11, 324-336. http://dx.doi.org/10.1016/0167-2789(84)90014-9

[10] Abdulloev, Kh.O., Bogolubsky, I.L. and Makhankov, V.G. (1974) One More Example of Inelastic Soliton Interaction. Physics Letters A, 56, 427-428. http://dx.doi.org/10.1016/0375-9601(76)90714-3

[11] Gardner, L.R.T., Gardner, G.A. and Geyikli, T. (1994) The Boundary Forced MKdV Equation. Journal of Computational Physics, 113, 5-12. http://dx.doi.org/10.1006/jcph.1994.1113

[12] Geyikli, T. and Karakoç, S.B.G. (2011) Septic B-Spline Collocation Method for the Numerical Solution of the Modified Equal Width Wave Equation. Applied Mathematics, 2, 739-749. http://dx.doi.org/10.4236/am.2011.26098

[13] Geyikli, T. and Karakoç, S.B.G. (2012) Petrov-Galerkin Method with Cubic B Splines for Solving the MEW Equation. Bulletin of the Belgian Mathematical Society, 19, 215-227.

[14] Esen, A. (2005) A Numerical Solution of the Equal Width Wave Equation by a Lumped Galerkin Method. Applied Mathematics and Computation, 168, 270-282. http://dx.doi.org/10.1016/j.amc.2004.08.013

[15] Esen, A. (2006) A Lumped Galerkin Method for the Numerical Solution of the Modified Equal-Width Wave Equation Using Quadratic B-Splines. International Journal of Computer Mathematics, 83, 449-459. http://dx.doi.org/10.1080/00207160600909918

[16] Saka, B. (2007) Algorithms for Numerical Solution of the Modified Equal Width Wave Equation Using Collocation Method. Mathematical and Computer Modelling, 45, 1096-1117. http://dx.doi.org/10.1016/j.mcm.2006.09.012

[17] Zaki, S.I. (2000) Solitary Wave Interactions for the Modified Equal Width Equation. Computer Physics Communications, 126, 219-231. http://dx.doi.org/10.1016/S0010-4655(99)00471-3

[18] Zaki, S.I. (2000) Least-Squares Finite Element Scheme for the EW Equation. Computer Methods in Applied Mechanics and Engineering, 189, 587-594. http://dx.doi.org/10.1016/S0045-7825(99)00312-6

[19] Wazwaz, A.M. (2006) The Tanh and the Sine-Cosine Methods for a Reliable Treatment of the Modified Equal Width Equation and Its Variants. Communications in Nonlinear Science and Numerical Simulation, 11, 148-160. http://dx.doi.org/10.1016/j.cnsns.2004.07.001

[20] Saka, B. and Dağb, İ. (2007) Quartic B-Spline Collocation Method to the Numerical Solutions of the Burgers’ Equation. Chaos, Solitons \& Fractals, 32, 1125-1137. http://dx.doi.org/10.1016/j.chaos.2005.11.037

[21] Lu, J. (2009) He's Variational Iteration Method for the Modified Equal Width Equation. Chaos, Solitons \& Fractals, 39, 2102-2109. http://dx.doi.org/10.1016/j.chaos.2007.06.104

[22] Evans, D.J. and Raslan, K.R. (2005) Solitary Waves for the Generalized Equal Width (GEW) Equation. International Journal of Computer Mathematics, 82, 445-455. http://dx.doi.org/10.1080/0020716042000272539

[23] Hamdi, S.W., Enright, H., Schiesser, W.E. and Gottlieb, J.J. (2003) Exact Solutions of the Generalized Equal Width Wave Equation. In: Kumar, V., Gavrilova, M.L., Tan, C.J.K. and L’Ecuyer, P., Eds., Computational Science and Its Application-ICCSA 2003, Springer, Berlin, 725-734. http://dx.doi.org/10.1007/3-540-44843-8 79

[24] Esen, A. and Kutluay, S. (2008) Solitary Wave Solutions of the Modified Equal Width Wave Equation. Communications in Nonlinear Science and Numerical Simulation, 13, 1538-1546. http://dx.doi.org/10.1016/j.cnsns.2006.09.018

[25] Karakoç, S.B.G. and Geyikli, T. (2012) Numerical Solution of the Modified Equal Width Wave Equation. International Journal of Differential Equations, 2012, Article ID: 587208. http://dx.doi.org/10.1155/2012/587208 